\title{
ANALYSIS OF MULTISTABLE VARIABLE STIFFNESS COMPOSITE PLATES
}

\author{
C. S. Sousa ${ }^{1}$, P. P. Camanho ${ }^{3}$, A. Suleman ${ }^{2}$ \\ ${ }^{1}$ Department of Mechanical Engineering, University of Porto \\ (corresponding-carlos.santos.sousa@fe.up.pt) \\ ${ }^{2}$ Department of Mechanical Engineering, University of Porto \\ ${ }^{3}$ Department of Mechanical Engineering, Instituto Superior Técnico - Technical University of Lisbon
}

\begin{abstract}
This paper presents a new concept for morphing composite structures based on variable stiffness composite plates. The variable stiffness morphing laminate proposed in this paper consists in a modified version of a straight fiber laminate composed of two regions, one with symmetric and the other with unsymmetric stacking sequence. Since there is a lay-up mismatch where the two regions meet, stress concentrations are expected to occur. A solution to ameliorate this effect is analyzed in which the fibers are allowed to vary smoothly along the plane from one region to the other. The particular trajectories followed by the curved fibers were designed such that the plate can be manufactured using Advanced Fiber Placement technology (AFP).

A finite element analysis of the laminate is performed to predict its out-of-plane displacements for the two possible stable configurations that may be obtained after the curing process. Then, the plate may be snapped from one shape to the other with the application of a force. This snap-through behavior is analyzed and compared with the original straight fiber plate.

The concept of a bi-stable Variable Stiffness Plate composed of regions of symmetric and unsymmetric lay-ups that preserve the tangential continuity of the fibers could be of great importance in morphing or shape-adaptable structures, such as winglets or flaps.
\end{abstract}

Keywords: Bi-stable composites, Unsymmetric composites, Variable Stiffness Panels, Morphing Structures.

\section{INTRODUCTION}

Multistable composite structures are characterized by having multiple equilibrium configurations, where each configuration represents a different geometric shape. Multistability in thin unsymmetric cross-ply laminates may result from residual stresses that build-up during the curing process or due to Gaussian curvature effects. In addition, the shape that is obtained after cure can be snapped into another stable shape with the application of a force or moment. Multistable composite laminates belong to a more general family of materials known as multifunctional composite materials, [1].

In the field of aerospace engineering, multistability is often combined with the properties of fiber-reinforced composite laminates not only because of the higher stiffness, higher strength and lower weight that these materials exhibit relative to conventional isotropic materials but also because of the possibility to taylor their stiffness to better adapt to different stress states. The combination of this properties make multistable fiber-reinforced composite laminates good candidates for morphing structures for aerospace applications. In this context, a morphing structure may be defined as a structure that is capable of modifying its macroscopic shape or dimensions in order to change its interaction with 
the surrounding environment or modify its operating conditions, [2,3]. A morphing structure is also considered to be a single deformable entity, with no relative movement between discrete parts [4].

A possible application for multistable laminated composites is aircraft wings, where morphing structures may replace conventional actuators and/or mechanically driven control surfaces, [3, 5]. Moreover, these structures may increase the performance of the aircraft in a way that cannot be achieved by conventional control surfaces since they provide lighter and more flexible solutions for locally adapting the shape of the wing according to the specific flight conditions [4]. A typical way of effectively morph wing-like structures using multistable laminated composites consists in varying the shape of its airfoil sections, [6]-8]. It is also possible to modify the wing span through the incorporation of a morphing winglet or varying the wing sweep angle, [8], although the later solution may be difficult to implement in large scale aircraft due to the higher loads involved. Another possibility is to modify the wing twist, [9. 10].

The range of applicability of morphing structures extends naturally to every surface that interacts with the surrounding environment. This includes helicopter rotor blades, airplane and compound helicopter propellers, wind turbine blades [9], aerodynamic fairings and ship hulls. Other applications include actuators for aerospace systems.

In order to change its shape, multistable laminated composites need the actuation of an external force. A typical solution is to embed piezoelectric layers into the structure or bond piezoelectric patches into its surface. Piezoelectric composite laminates have the ability to change its geometry when electric energy is supplied to the piezoelectric system. Due to the bistability of thin unsymmetric laminates, they have the advantage of requiring energy only to trigger the snap-through between one stable shape to the other, since either configuration can be maintained onwards. Multistable laminated composites actuated by piezoelectric patches were investigated by Portela, et al. [11]. Many analytical and numerical models have been developed for the design of general morphing laminated composites with simple geometric shapes that use piezoelectric actuation. A thorough literature review is given by Correia, et al. [12].

This paper is focused in bistable fiber-reinforced laminated composites and introduces the key feature of variable stiffness through the use of curvilinear fiber plies. Since the laminate investigated here is in close relationship with the bistable laminate model of Mattioni, et al. [13, 14], a short description of this model is given. Before curing, the laminate consists of a flat rectangular composite laminate whose planform is comprised of two distinct and adjacent regions, with symmetric and unsymmetric stacking sequences respectively. Each region consists in a thin cross-ply laminate with a square shape. Thin square laminates of unsymmetric layup have the ability to develop large out-of-plane displacements and curvature when subjected to temperature variations. This behaviour is induced by differential thermal stresses that arise due to the mismatch of the thermal expansion properties between the matrix and the unidirectional fibers. Such temperature variations occur during the curing stage of the laminate. Hyer [15] investigated the cured shapes of this type of laminates and observed that they warp into a right circular cylinder instead of being saddle shaped, as predicted by Classic Lamination Theory. Moreover, another stable cylindrical shape could be obtained from the first with the action of a force, giving this type of laminates the property of bi-stability. Thus, in the model of Mattioni, et $a l$. , the region with unsymmetric sequence renders the structure bistable while the symmetric sequence region acts like a neighbouring interacting structure.

Efforts in predicting the multiple stable shapes and understanding the snap-through mechanism of thin unsymmetric laminates have been made by many researchers. Hamamoto and Hyer [16] and Dano and Hyer [17] extended Classic Laminate Theory in order to predict the room temperature shapes of square unsymmetric cross-ply laminates. This theory have been compared against numerical results and validated with experimental data.

After the multiple shapes have been obtained, it is important to know which forces need to be applied in order to change from one stable shape to the other and to understand how the structure behaves during this process. Analytical models for predicting the snap-through forces and moments were developed by Dano and Hyer [18, 19]. The models were based on the Rayleigh-Ritz method and 
virtual work principles and were compared against experimental data. More recently, Potter, et al. [20] investigated a 2-ply unsymmetric laminate as it was snapped from one stable shape to the other. Loads and deformations were monitored throughout the experiment and it was observed that the snap-through occurs in two distinct events, where one side of the plate snaps after the other. Pirrera, et al. [21] developed an analytical model based on the Rayleigh-Ritz method with higher order polynomials and predicted the multi-event snap-through observed by Potter.

Once the multiple shapes and forces involved for triggering snap-through are well understood, it is important to predict weather or not the structure will carry the induced stresses and strains and which values of the applied forces may lead to the onset of failure. In this context, it is expected that the abrupt change in direction of the fibers from one region to the other of a given layer in the model of Mattioni, et al. may yield a poor overall performance of the laminate due to stress concentrations in the boundary between these regions. This motivates a new design were curved fibers are applied to bridge the unsymmetric and symmetric regions. Suppose that in the original straight fiber model the fibers are oriented at $90^{\circ}$ in a given region and $0^{\circ}$ in the other. Then, in the new design the curvilinear fibers would start at $90^{\circ}$ and gradually change their trajectories to match the $0^{\circ}$ orientation angle of the other region.

The concept of continuously varying the angle of the fiber path as it traverses the plane of the lamina was introduced by Gürdal and Olmedo [22,23] and Olmedo and Gürdal [24] and has been the subject of thorough investigation. Since the orientation angle of the fiber is, in general, different from point to point in a lamina, it induces a local variation of the plate elastic properties leading to the concept of Variable Stiffness Panel(s) (VSP). Such advanced composite panels may be manufactured with Tow Placement Technology. A literature survey on tow placement technology and Variable Stiffness Panels is given by $W u[25]$.

Gürdal and Olmedo proposed a linear variation of the fiber angle along a given direction and obtained analytical and numerical solutions for simple plates. In this design, each tow could be defined in terms of a reference path that traverses the centerline of the tow. The reference path could be described in terms of three angles and additional paths could be placed by either the parallel or the shifted methods. The former is achieved by positioning each path parallel to the original reference path while the later is obtained if each new path is a copy of the original path but translated in a direction perpendicular to its axis.

A simplified Finite Element computational model was developed for the analysis of Variable Stiffness composite laminates in which the orientation of the fibers follows the formulation of Gürdal and Olmedo. The laminate is designed to have two distinct regions in the planform each with a different layup as in the model of Mattioni, et al.. The computational model is generated through an input script for use in the commercial software ABAQUS ${ }^{\circledR}[26]$.

The aim of this paper is to analyse the geometry of the curing shapes obtained with VSP morphing laminates as well as its stability properties. Furthermore, the ability of the finite element model to capture the snap-through behaviour of the variable stiffness laminate is also investigated. Then a failure criteria is introduced in order to predict the onset of failure and to access in which measure the smooth variation of fiber angle may lead to a better stress distribution and load carrying capability of the new design without compromising the bi-stable behaviour of the laminate.

\section{LAMINATE MODEL}

The composite laminates proposed in this paper are composed of curved fiber plies possibly interleaved with conventional straight fiber plies. The curvilinear plies belong to a more general family of laminae in which a curved fiber region is adjacent to a straight fiber region. Then, the plies are stacked such that one side of the laminate has an unsymmetric stacking sequence and the other side has a symmetric or, at least, approximately symmetric stacking sequence ${ }^{1}$. A typical ply is represented in

\footnotetext{
${ }^{1}$ In the sense that the stability characteristics of the true unsymmetric sequence do not significantly depart from an idealized symmetric stacking sequence.
} 
Fig. 1 .

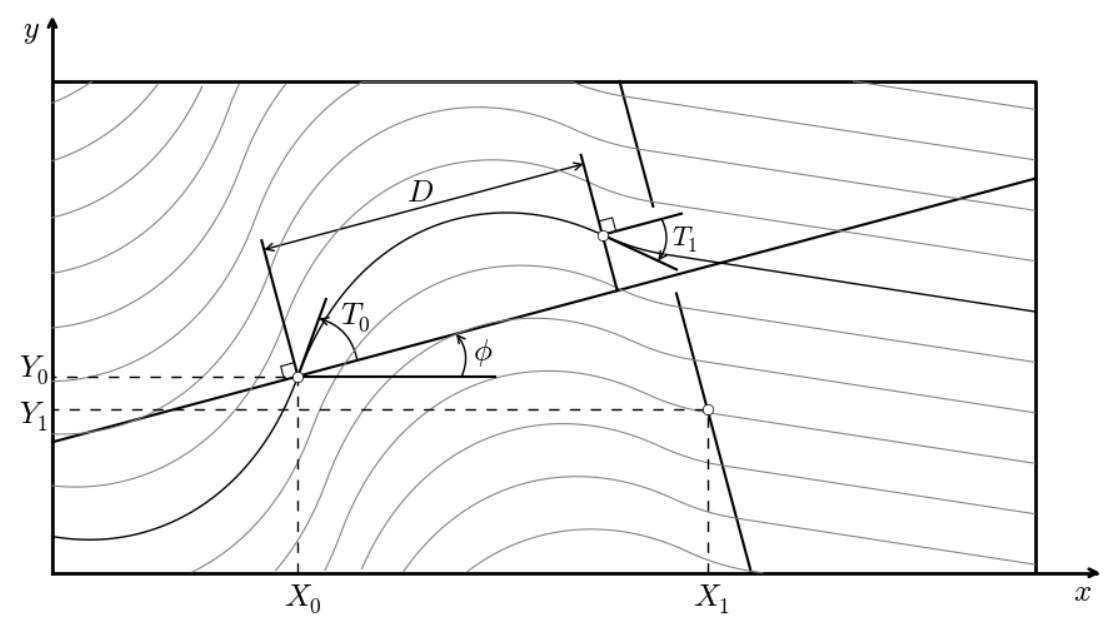

Figure 1. Sketch of a general ply.

It can be verified that the curvilinear fiber region may not have a square or rectangular shape depending on the parameter $\phi$. The fiber orientation angle of the straight fiber region is imposed by the geometric constraint that forces the fiber to preserve tangential continuity at the boundary that separates both regions.

To obtain a continuous distribution of fibers at every point of each ply, it is assumed that each tow is represented by a single fiber or, more rigorously, that each tow band is taken to the limit of zero width. The shifted method is adopted, in which every curved fiber contained in a ply can be obtained by a translation in the $x y$ plane of an arbitrary complete fiber. With these assumptions, neither the stepped termination pattern or the tow band overlaps and/or gaps will be modelled. Fig. 2 shows a schematic of the simplifications involved in the present plate model.
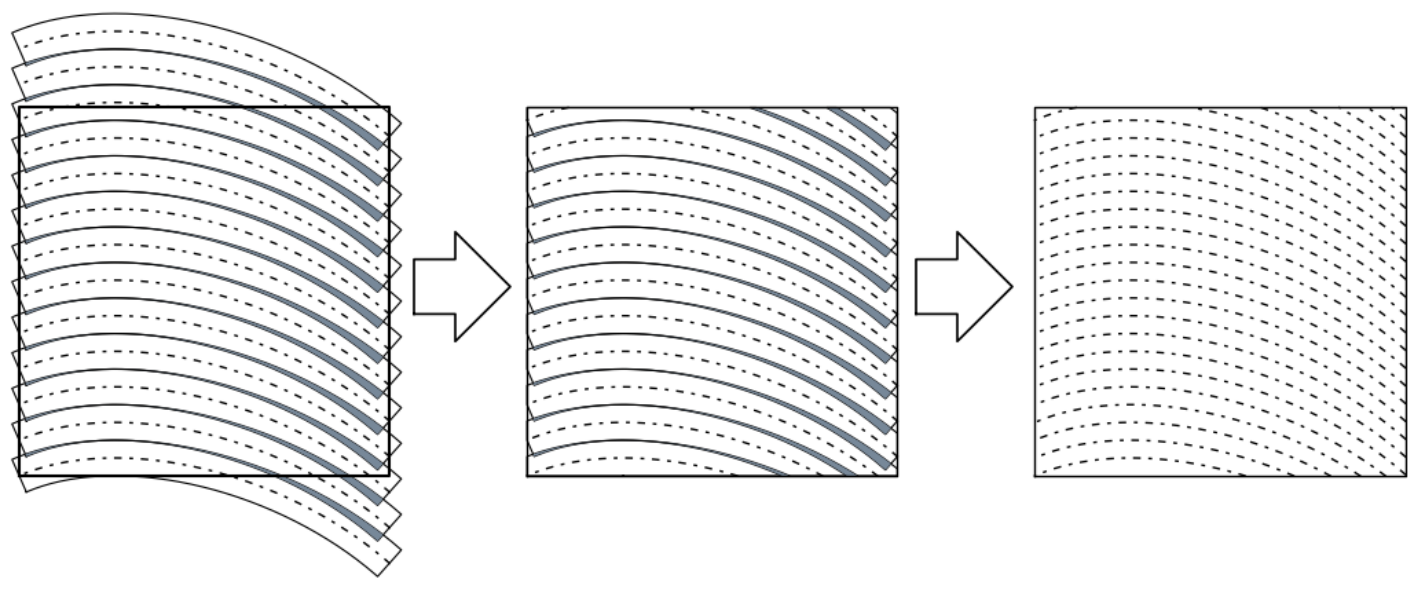

Figure 2. Schematic of the simplification steps in the planar distribution of curved fibers in the plane. Filled darker areas represent regions of tow band overlaps.

The variation of the fiber orientation angle $\psi$ along the fiber reference axis follows the formu- 
lation derived by Olmedo and Gürdal [24],

$$
\psi(r)= \begin{cases}T_{0}+\left(T_{1}-T_{0}\right) \frac{r}{D} & \text { if } 0 \leq r<D, \\ T_{0}-\left(T_{1}-T_{0}\right) \frac{r}{D} & \text { if }-D \leq r<0\end{cases}
$$

where $r$ is the distance measured along the reference axis and $T_{0}$ and $T_{1}$ are the fiber orientation angles at the origin and at $r=D$, respectively. The angles $T_{0}$ and $T_{1}$ are measured with respect to the reference axis and are depicted in Fig. 1. A clear and concise description of the reference fiber path as well as of the shifted and parallel methods is given by Waldhart [27].

It is possible to extend the domain of definition of $\psi$ by assuming it to be periodic and continuous, with period $2 D$. This can be stated mathematically by the function $\tilde{\psi}: \mathbb{R} \rightarrow \mathbb{R}$

$$
\tilde{\psi}(r)=\psi\left(r-2 D\left\lfloor\frac{r}{2 D}+\frac{1}{2}\right\rfloor\right)
$$

where $\lfloor x\rfloor$ represents the floor operator defined by

$$
\lfloor x\rfloor=\max \{m \in \mathbb{Z} \mid m \leq x\}
$$

Function (1) can be integrated to obtain the trajectory of the fiber with respect to the reference axis,

$$
g(r)=\left\{\begin{array}{cl}
-\frac{D}{T_{1}-T_{0}} \log \frac{\cos \psi(r)}{\cos \left(T_{0}\right)} & \text { if } 0 \leq r<D, \\
\frac{D}{T_{1}-T_{0}} \log \frac{\cos \psi(r)}{\cos \left(T_{0}\right)} & \text { if }-D \leq r<0
\end{array}\right.
$$

The derived function $\tilde{g}$ is constructed from $g$ by requiring it to be periodic and continuous,

$$
\tilde{g}(r)=g\left(r-2 D\left\lfloor\frac{r}{2 D}+\frac{1}{2}\right\rfloor\right)-2 g(-D)
$$

For a more general fiber trajectory the reference axis can be rotated by an angle $\phi$, and then translated by a vector $\mathbf{v}=X_{0} \mathbf{e}_{x}+Y_{0} \mathbf{e}_{y}$. The rotated frame coordinates are related to the coordinate along the reference fiber axis, $r$, and to the function $\tilde{g}$ by

$$
\begin{aligned}
& x=r \cos \phi-\tilde{g}(r) \sin \phi+X_{0} \\
& y=r \sin \phi+\tilde{g}(r) \cos \phi+Y_{0}
\end{aligned}
$$

where $\phi$ is the angle between the horizontal direction and the fiber reference axis.

\subsection{THE STRAIGHT-FIBER MODEL}

Mattioni, et al. [13, 14] analysed piecewise-continuous plate models for different cases of planar dimensions and number of layers. The $180 \mathrm{~mm}$ high by $360 \mathrm{~mm}$ wide, four layered plate serve as a basis for the VSP model analysed in the current paper. It consists in a half symmetric, half unsymmetric rectangular composite laminate. Each half can be regarded as a square plate and is composed of four $0.125 \mathrm{~mm}$ thick plies made of unidirectional T300-914 carbon fiber composite which properties are listed in Table 1 . The symmetric and unsymmetric regions have stacking sequences given by $[0 / 90]_{S}$ and $\left[0_{2} / 90_{2}\right]_{T}$, respectively. 
Table 1. Material properties of unidirectional T300-914 carbon fiber/epoxy composite.

\begin{tabular}{lc}
\hline Property & T300-914 \\
\hline$E_{11}(\mathrm{GPa})$ & 130 \\
$E_{22}(\mathrm{GPa})$ & 10 \\
$G_{12}(\mathrm{GPa})$ & 4.4 \\
$\nu_{12}$ & 0.3 \\
$\alpha_{1}\left(1 /{ }^{\circ} \mathrm{C}\right)$ & $-1.8 \times 10^{-8}$ \\
$\alpha_{2}\left(1 /{ }^{\circ} \mathrm{C}\right)$ & $30 \times 10^{-6}$ \\
\hline
\end{tabular}

\subsection{THE VSP MODEL}

A new Variable Stiffness laminate model is developed that exploits the properties of the bistable model of Mattioni, et al. [13, 14], but uses the general curvilinear-fiber ply introduced previously. There are several strategies that may be adopted for the design of the general curvilinear-fiber ply in order to arrive to a configuration where one side of the laminate is bi-stable while the other acts as an interacting structure. It is clear that, the bi-stable part should have an unsymmetric stacking sequence in order to develop curvature at the curing stage. In addition, the interacting structure should have a stacking sequence as close to symmetric as possible to restrain it from developing curvature.

According to this concept, a possible strategy to develop a Variable Stiffness laminate is illustrated in Fig. 3 a, where the fiber orientation varies from an initially vertical orientation at the right-hand side to a near horizontal direction at free-edge at the left-hand side the ply. Fig. 3a was obtained by choosing the appropriate combination of angles defined in Fig. 1, namely the reference axis angle, $\phi$, initial angle, $T_{0}$, and final angle $T_{1}$. However, for this value of the angle $\phi$, the fiber orientation angle is only exactly $0^{\circ}$ at the lower left corner and increases along the free-edge of the left-hand side, which may not be desired. Also, the boundary between the straight and curvilinear fibers is highly inclined which will result in a large region with mixed symmetric and unsymmetric stacking sequences after the remaining unidirectional plies are included. By decreasing the reference axis angle, Fig. $3 \mathrm{~b}$, the range of variation of the fiber orientation angles at the free-edge of the left-hand side decreases but at the cost of a higher concentration of fibers at the interface between the symmetric and unsymmetric regions. This may not be desired since the abrupt change in fiber density may result in strong localized stress gradients near the interface.
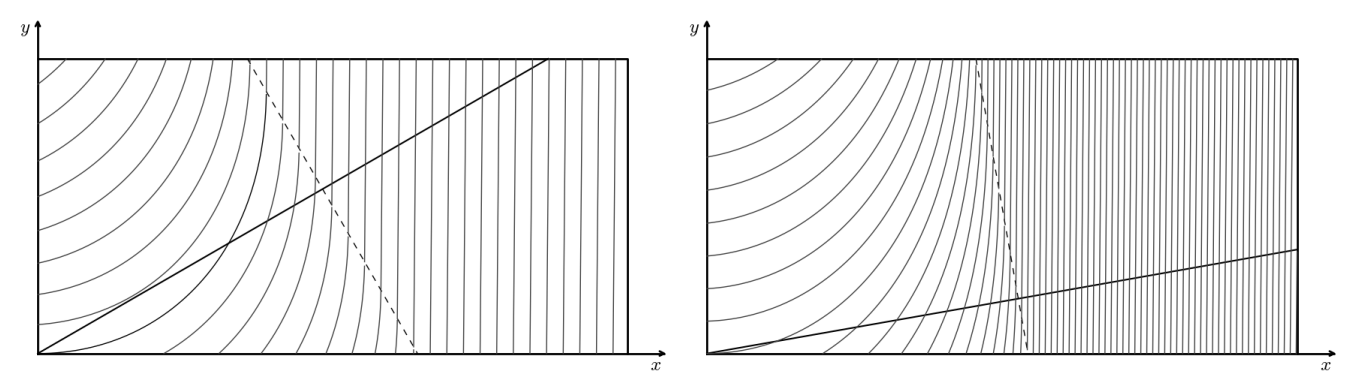

Figure 3. Variable Stiffness morphing laminate based on curvilinear fibers. a) Higher angle of the reference fiber axis, $\phi=30^{\circ}$. b) Lower angle of the reference fiber axis, $\phi=10^{\circ}$

A different strategy is adopted in this paper, in which the transition from an horizontal to a vertical orientation angle established using curvilinear-fiber plies occurs in the interacting structure, 
thus destroying the symmetry condition of the stacking sequence. The full stacking sequence of a four-layer variable stiffness laminate that implements this strategy is represented in Fig. 4a. Note that the region at the right-hand side of the laminate can only be considered approximately symmetric due to the introduction of the second curvilinear-fiber ply, as compared to the truly symmetric region of the right-hand side of the laminate model of Mattioni et al., represented at Fig. 4p. However, in order to establish an analogy between the straight-fiber laminate model of Mattioni, et al. and the Variable Stiffness morphing laminate proposed in this paper, the unsymmetric region of the right-hand side of the variable stiffness laminate, illustrated in Fig. 44, will henceforth be denoted as 'symmetric region'.

The Variable Stiffness laminate represented in Fig. 4a is composed of two curvilinear-fiber plies interleaved by two unidirectional plies. The configuration of the reference fiber in the top curvilinear-fiber ply is defined in terms of the parameters of Fig. 11, which are assigned with the values listed in Table 2 .

The characteristic length in the direction of the reference axis, $D$, is such that the angle $T_{1}$ is specified at the boundary line between the symmetric and unsymmetric regions, represented by a dashed line in the second and fourth plies in Fig. 4 a. Hence, the orientation angle of the straight fibers is directly given by $\phi+T_{1}$. This yields the following relation for $D$,

$$
D=\left(X_{1}-X_{0}\right) \cos \phi+\left(Y_{1}-Y_{0}\right) \sin \phi
$$

In order to obtain a smooth transition from an orientation angle of $0^{\circ}$ to $90^{\circ}$ several combinations of $\phi\left\langle T_{0} \mid T_{1}\right\rangle$ can be adopted. If the inclination angle of the reference axis is $0^{\circ}$, then the required orientation angle of the reference fiber at the origin point, $T_{0}$, would be $90^{\circ}$. However, the function $\tilde{g}$ that describes the reference fiber path would be indeterminate in this case. Hence, an angle of $\phi=10^{\circ}$ is adopted for the variable stiffness plies.

Table 2. Parameters for the reference path of the VSP model.

\begin{tabular}{ccccccccc}
\hline Width, $w(\mathrm{~mm})$ & Height, $h(\mathrm{~mm})$ & $X_{0}$ & $Y_{0}$ & $X_{1}$ & $Y_{1}$ & $\phi$ & $T_{0}$ & $T_{1}$ \\
\hline 360 & 180 & 0 & 0 & $w / 2$ & $h / 2$ & $10^{\circ}$ & $80^{\circ}$ & $-10^{\circ}$
\end{tabular}

\subsection{FEM Model}

The VSP model is analysed using the Finite Element Method implemented in ABAQUS ${ }^{\circledR}$ [26]. The plate is discretized into 800 linear quadrilateral elements of type S4R, as depicted in Fig. 5. For the curvilinear regions the orientation angle is computed at the centroid of each element with the function $\tilde{\psi}$ given by equation (2). The coordinates of the centroid are conveniently transformed through the transformation given by (5).

For the simulation of the curing process the plate is allowed to cool-down to room temperature completely unrestrained. To avoid rigid body motion, the plate is clamped at its geometric center.

After reaching an equilibrium state the snap-through analysis is performed, where the transition between one stable shape to the other is simulated. The clamped condition is removed and the four corner nodes become free to move in the $x y$ plane. In addition, the geometric center of the unsymmetric half of the plate is constrained to move along the $z$ direction. To suppress rigid body rotation, this node is also restrained from rotating around the $z$ axis. For the static analysis with artificial damping a vertical concentrated force is applied at this node. 


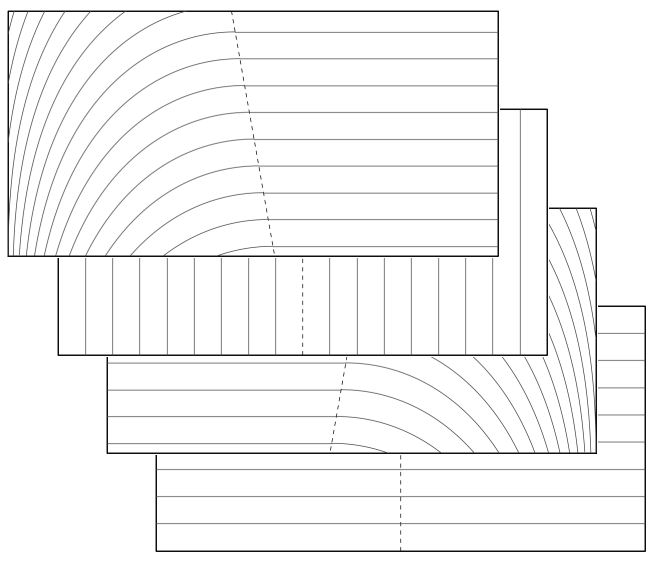

a)

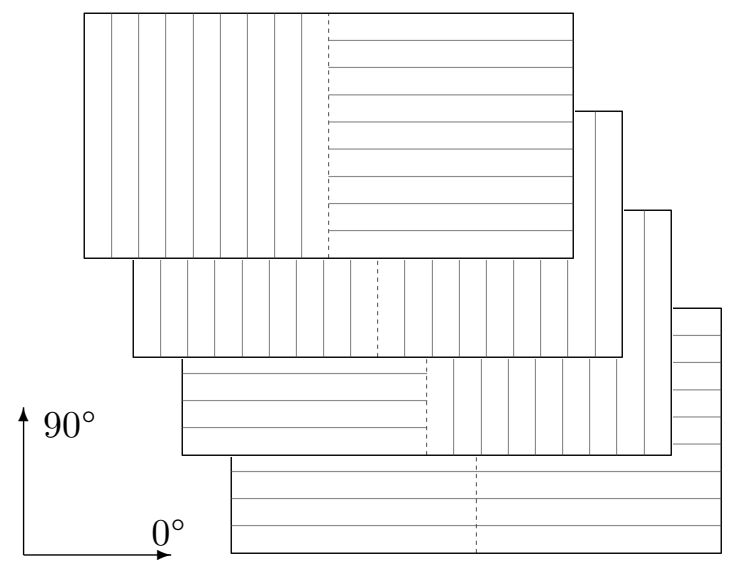

b)

Figure 4. a) Variable Stiffness morphing laminate based on curvilinear fibers. b) Laminate model of Mattioni, et al.

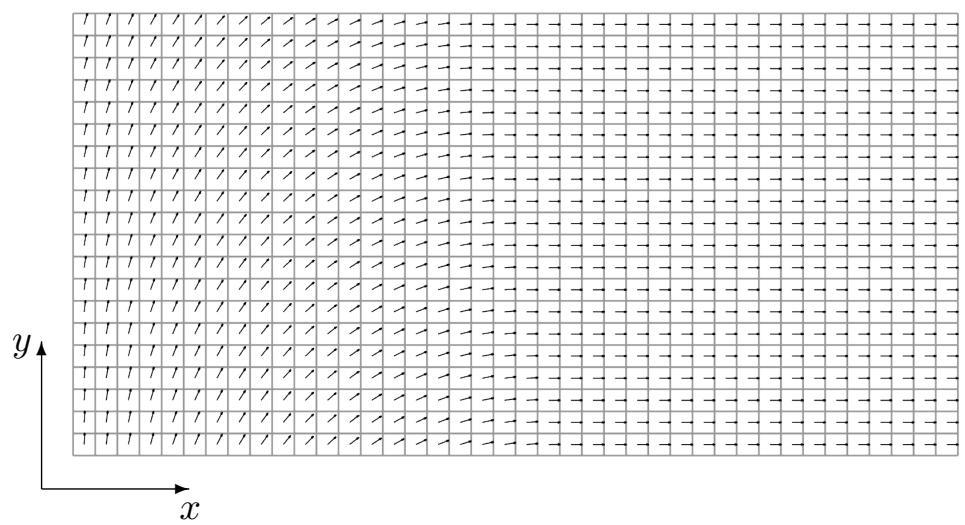

Figure 5. Discretized domain for ply 4 of the VSP model showing the fiber orientation for each element.

\section{ANALYSIS OF THE COOL-DOWN PROCESS}

An important stage of the manufacture of carbon/epoxy composite laminates is the curing process. First the plate is subjected to a gradually increasing uniform temperature field until a maximum specified value. This stage is characterized by the crosslinking of viscous resin and fibers and the material properties vary in a complex manner. After the maximum temperature has been reached, the plate is allowed to cool-down to room temperature. During this second stage, the material properties are not influenced by chemical activity and can be described through pure mechanical analysis [28]. Furthermore, Hyer [15, 29] observed that unsymmetric thin cross-ply laminates may develop into a saddle or cylindrical shape due to the lower expansion rate of the material in the fiber direction relative to a direction perpendicular to the fibers.

In this study, only the cool-down process is simulated. It is assumed that the plate is stress-free at the beginning of this stage. For the purpose of this paper, viscoelastic effects and moisture absorption will be neglected.

Since the plate presents multiple stable shapes, the equilibrium path is characterized by limit points and bifurcation. Non-linear static implicit methods formulated in terms of a tangent stiffness 
matrix may fail to converge close to limit points. If a limit point is passed, the tangent stiffness matrix will have negative eigenvalues and the structure will be in a state of unstable equilibrium. For the cooldown process, the problem may be assumed to be quasi-static and a fictitious damping matrix may be assigned to the structure. In ABAQUS/Standard ${ }^{\circledR}$, this is implemented through the viscous force vector,

$$
F_{v}=c \mathbf{M}^{*} v
$$

where $\mathbf{M}^{*}$ is the artificial mass matrix, $c$ is a damping factor and $v$ is the vector of nodal velocities. The global equilibrium equations become,

$$
P-I-F_{v}=0
$$

where $P$ and $I$ are the vectors of external and internal nodal forces, respectively.

The value of the damping factor should be high enough to damp local instabilities and small enough to ensure the accuracy of the solution. For the cool-down process, a value of $c=2.5 \times 10^{-7}$ is used in order to replicate the simulation of Mattioni, et al. [13]. Initially, the temperature is uniformly distributed along the flat plate at $140^{\circ}$. The plate is then allowed to cool until its temperature reaches $0^{\circ}$. The resulting shapes for both straight and curvilinear fiber plate models is depicted in Fig. 6 .

a)

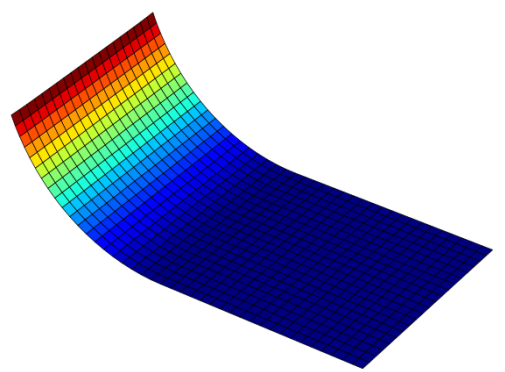

Figure 6. Perspective view of deformed plate after cool-down for $\Delta \mathrm{T}=-140{ }^{\circ} \mathrm{C}$. The color map represents the height of each point in the surface. a) Straight fiber model. b) Curvilinear fiber model.

The room-temperature shape of the straight fiber model presents curvature in the unsymmetric half but remains flat in the symmetric half. The curled shape thus formed is identified as shape 1 in the work of Mattioni, et al. and is the natural stable shape for this plate model.

The curvilinear model, however, shows a different tendency for its stable shape after cool-down which is comparable to the second stable shape of the straight fiber model.

Fig. 7 shows the variation of principal curvatures measured at the center of the unsymmetric region with temperature. The sign convention used is that curvature is positive when the center of curvature is in the negative normal vector direction. The normal vector to the surface is initially pointing in the positive $z$-direction.

The principal curvatures of the straight fiber model are nonlinear up to about $130{ }^{\circ} \mathrm{C}$, where the unsymmetric region is deformed into a saddle shape. After this point the principal curvatures vary linearly with temperature, as predicted by classic laminate theory. For the curvilinear fiber model, the saddle shape is obtained at a lower temperature of about $124{ }^{\circ} \mathrm{C}$. After this point, the variation of the major principal curvature, $K_{1}$, with temperature is not exactly linear presenting a slight reduction in slope as temperature decreases.

\section{SIMULATION OF SNAP-THROUGH}

The curing stage is completed once the plate has reached a state of equilibrium at roomtemperature. The shape obtained in this fashion corresponds to one of its stable shapes. The plate may then be snapped into its second stable shape by applying a transverse force or bending moment. 

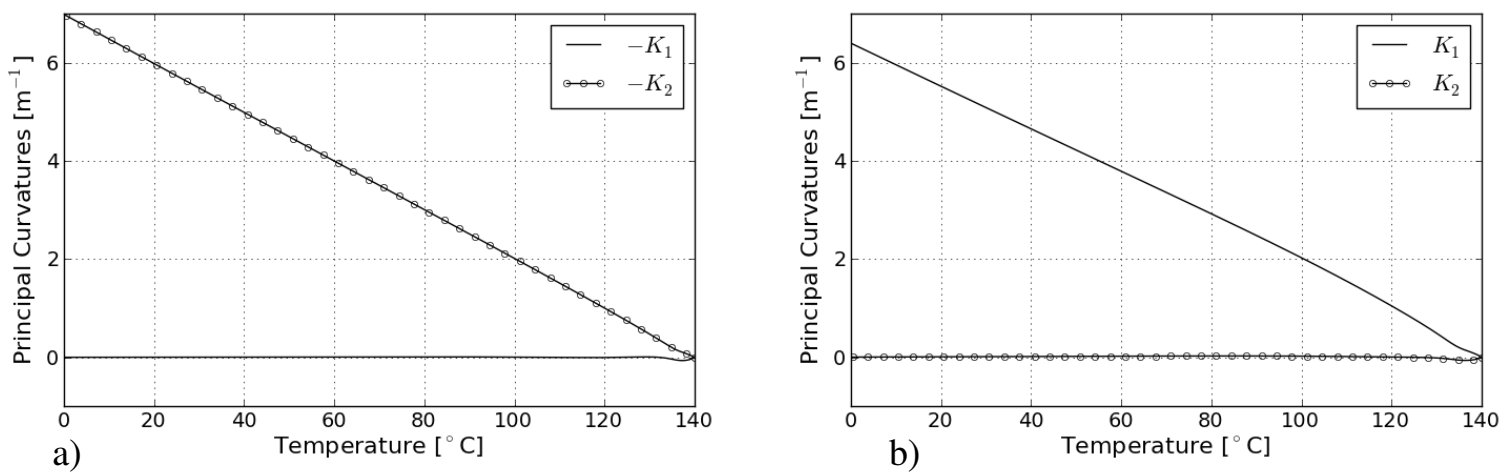

Figure 7. Variation of principal curvatures at center of unsymmetric region during cool-down. a) Straight fiber model. b) Curvilinear fiber model.

The snap-through mechanism is inherently a dynamic process since the magnitude of viscous forces is significant during the unstable transition from one equilibrium shape into the other. The viscous relaxation method used to analyse the cool-down process is not able to track the elastic response of the structure past limit points. In order to track static equilibrium states after this points the modified Riks method is adopted [30,31]. In this method the applied load magnitude becomes a parameter that is solved together with the displacement.

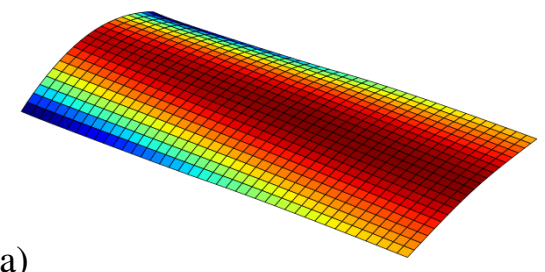

a)

Figure 8. Perspective view of the plate models after snap-through when no external forces are applied. The color map represents the height of each point in the surface. a) Second shape of the straight-fiber model. b) First shape of the curvilinear fiber model.

Fig. 8 shows a perspective view of the deformed geometries of the plates that were obtained after the snap-through and after removing externally applied loads. Fig. $8 \mathrm{a}$ and $8 \mathrm{p}$ correspond to the second shape of the straight fiber model and to the first shape of the curvilinear fiber model, respectively and may be compared with the corresponding plots of Fig. 7. It is observed that the second shape of both models is similar, although the curvature of the free-edges at $x=0$ and at $x=360 \mathrm{~mm}$ is higher in the curvilinear model, as depicted in Fig. 9. This is the result of a loss of stiffness in the $y$ direction of the curvilinear relative to the straight fiber model. Rather than being oriented strictly at $90^{\circ}$, the fibers of the former model now vary continuously in the range from $0^{\circ}$ to $90^{\circ}$.

The resultant geometry of the curvilinear model shown in Fig. $8 \mathrm{p}$ is also twisted along its longitudinal direction. Consider a plane passing through the geometric center of the plate and parallel to $x y$ plane. From Fig. $4 \mathrm{a}$ it is clear that the curvilinear fiber trajectories are not symmetric with respect to this plane and, as an effect, complex tensile-shear interactions occur. In order to remove this effect the plate should be balanced with plies with equal trajectories but mirrored about this plane. 

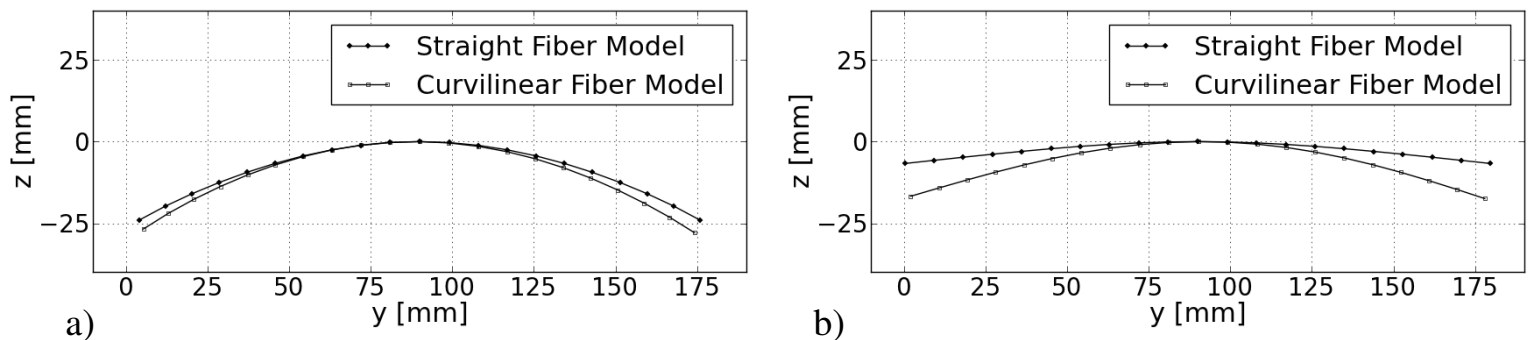

Figure 9. Comparison of the free-edges of the second shape between the straight and curvilinear fiber models. a) Free-edge at $x=0$. b) Free-edge at $x=360 \mathrm{~mm}$.

Fig. 10 shows how the applied force and the total reaction force at the four corner nodes varies with displacement. The plot of Fig. 10a represents the snap-through of the straight fiber model from the first to the second shape while the plot of Fig. $10 \mathrm{~b}$ refers to the curvilinear fiber model but from the second to the first shape. It can be verified that past the first critical point, label $a$ in Fig. $10 \mathrm{p}$, the structure is damped through viscous forces to counteract its sudden decrease in stiffness. The applied load is monotonically increasing since the viscous forces are high enough to ensure static equilibrium during unstable transitions of the structure.

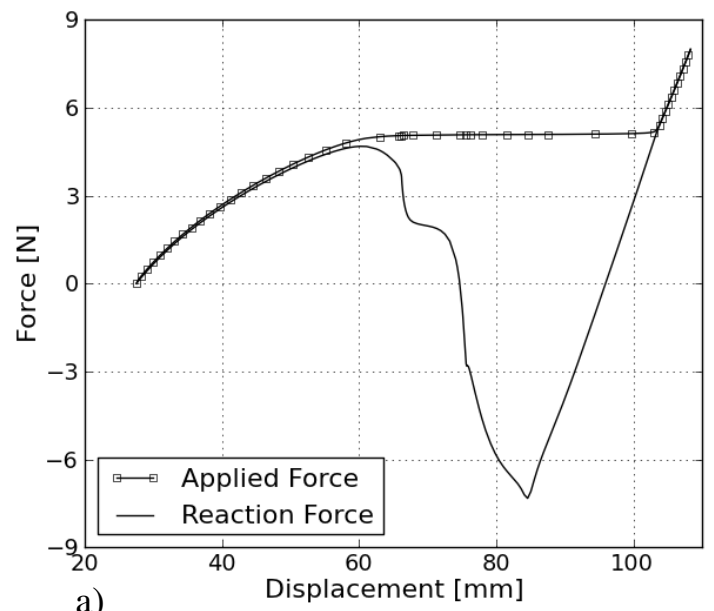

a)

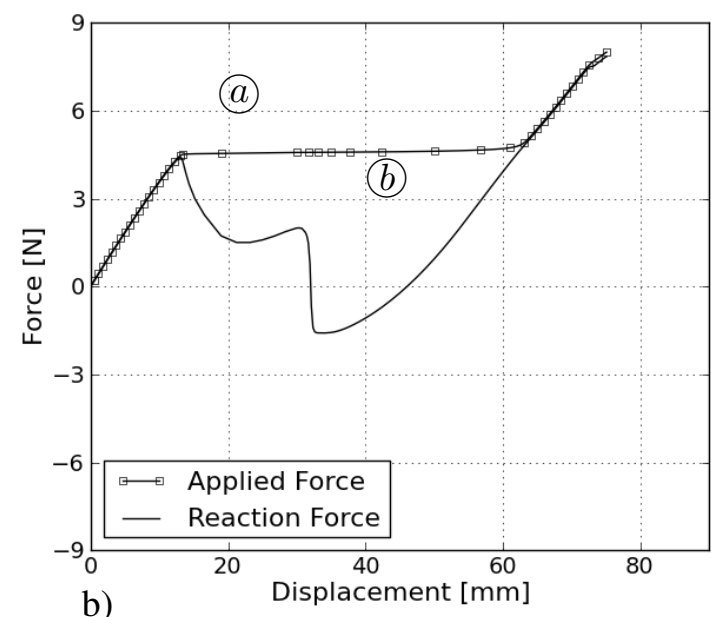

b)

Figure 10. Variation of the applied force and total reaction force with vertical displacement during the snap-through. a) Straight fiber model. b) Curvilinear fiber model.

The snap-through of both plate models does not occur in a single event. For the straight fiber model the reaction load decreases slowly after the limit point and then drops suddenly which corresponds to a reversal in the curvature of one side of the unsymmetric region. This sudden drop stops at about half of the critical load as the slope of the force/displacement curve increases. Then, a second drop in the reaction force is observed as the curvature of the second half of the unsymmetric region is reversed.

The snap-through of the curvilinear fiber model also occurs in two distinct events as shown in the plot of Fig. 10b. It is verified that the reaction force falls rapidly after the first critical point. This is identified as a cusp in the reaction force/displacement curve at point $a$, which is not verified if the plate were to change from the first to the second shape. After this point the slope of the curve becomes 
negative and slowly increases to a positive value. The plate is actually recovering some stiffness while one half of the unsymmetric region is developing curvature and the other is flattening, as shown in Fig. 18. Shortly after point $b$

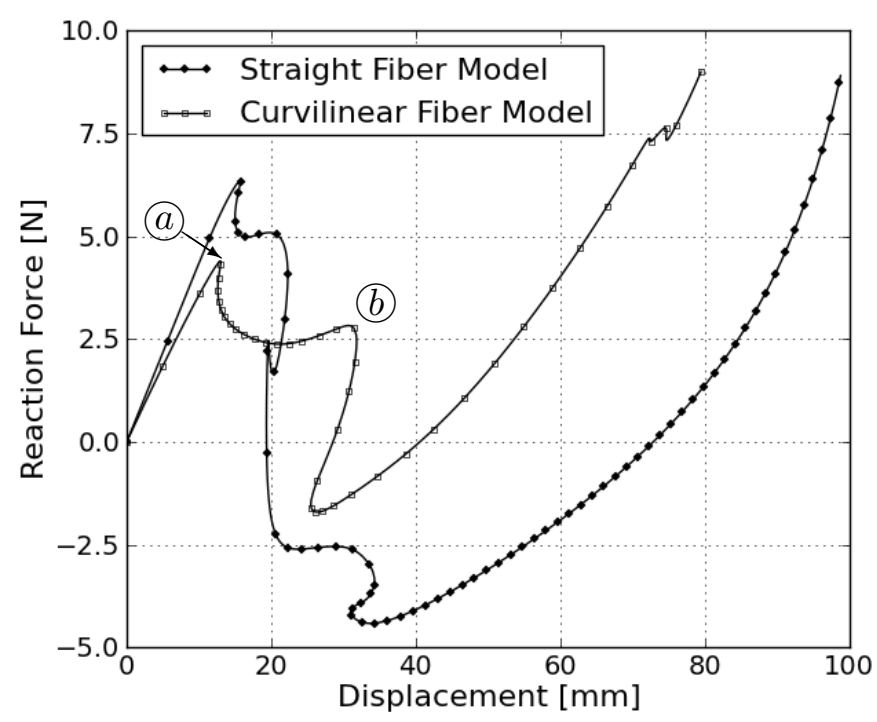

Figure 11. Variation of the reaction force with displacement obtained with the modified Riks method. Comparison between the straight and curvilinear fiber models.

Fig. 11 shows the variation of the reaction force with displacement using the modified Riks method. In this plot, both plate models experience snap-through from the second to the first shape. The force and displacement are measured at the center of the unsymmetric region of the laminates and the displacement is measured along the vertical axis and relative to the deformed geometry at the start of the snap-through step. The curve corresponding to the curvilinear fiber model may be compared against Fig. 10p. The static method using stabilization yields the same result for the magnitude of the force necessary to activate the snap-through at point $a$, but underpredicts the value of the force at point $b$ by $29 \%$ relative to the modified Riks method.

Since the modified Riks method is essentialy an elastic model, the curve with respect to the straight fiber plate plotted in Fig. 11 may also be compared to Fig. 10a. This is possible if the force and displacement scales are inverted in the former plot and the displacement under consideration ranges from 19 to $72 \mathrm{~mm}$. The value of the applied force required for snap-through that is obtained from the static method with viscous damping is the same value reported by Mattioni, et al. [13]. However this value may be sensible to variations in the damping factor that is specified prior to the computations.

The reaction force, however, represents the force required to maintain the static equilibrium and its value at the critical point is closer from the experimental result, as can be seen in Table 4 The Riks method slightly underpredicts the experimental value by $5 \%$. Table 3 lists the values for the critical loads obtained using this method.

Table 3. Magnitude of the critical loads required for the snap-through obtained by the modified Riks method. Values are given in $\mathrm{N}$.

\begin{tabular}{lccc}
\hline Direction & Straight Fibers & Curvilinear Fibers & \% Difference \\
\hline $1^{\text {st }} \rightarrow 2^{\text {nd }}$ & 4.41 & 1.71 & 61.2 \\
$2^{\text {nd }} \rightarrow 1^{\text {st }}$ & 6.33 & 4.39 & 30.6 \\
\hline
\end{tabular}


Table 4. Magnitude of the critical loads required for the snap-through of the straight fiber model from the first to the second shape. Values are given in $\mathrm{N}$.

\begin{tabular}{ccc}
\hline Applied & Reaction & Experimental $^{1}$ \\
\hline 4.92 & 4.69 & 4.65 \\
\hline From reference & 13 & \\
\end{tabular}

\section{ANALYSIS OF FIRST-PLY FAILURE}

During the snap-through stage the plate may be subjected to high localized stresses that may ultimately lead to failure of the material. In this section, the onset of failure is predicted with a criteria developed at NASA - Langley Research Centre (LaRC) [32, 33]. It consists in a physically-based criteria based on the components of the stress tensor and in-situ strengths of the material which may accurately predict the main failure modes of fiber reinforced laminated composites: matrix tensile failure, matrix compression failure, fiber tensile failure, fiber compressive failure. In this paper it is assumed that the first ply failure is mainly dependent of the membrane components of the stress tensor during the snap-through stage. If the plate is subjected to an increasing applied load after the snap-through process then this assumption may not be valid. With this assumption, the LaRC03 version of the mentioned criteria is used, which does not take into account the transverse shear stress components of the stress tensor, $\sigma_{13}$ and $\sigma_{23}$. This is also in agreement with the shell model adopted. As consequence, this criteria is not capable of predicting delamination which typicaly occurs due to interlaminar stresses. The formulation of the $\mathrm{LaRC} 03$ criteria is given in appendix $\mathrm{B}$.

The LaRC03 criteria is implemented in ABAQUS ${ }^{\circledR}$ through a FORTRAN subroutine known as User defined VARiables at finite element Material (UVARM). This subroutine is run at every load increment and for every integration point of the Finite Element model and outputs the LaRC failure indices at every element and for every layer of the laminate.

Table 5 shows the value of the maximum transverse tensile and longitudinal tensile failure indices measured in the straight-fiber model. The maximum values were computed over the entire snap-through step and for the four layers of the laminate. In order to pinpoint the location of this maximum value in Fig. 10a, values for the total reaction force at the four corners nodes and for the vertical displacement of the center of the unsymmetric region of the laminate are also listed. Again, displacement is measured relative to the deformed geometry at the start of the snap-through step, when no external forces are applied. It can be verified that both transverse tensile and longitudinal tensile stresses predicted using the LaRC03 criteria are below unity for every load increment of the snapthrough step. Also, the transverse and longitudinal compression failure indices are everywhere zero. The higher value observed for the transverse tensile failure index corresponds to the beginning of the snap-through process and so is a result of the residual thermal stresses that originated from the cure stage. It is verified that the value of the transverse tensile failure is about 8 times higher than the value of the longitudinal tensile failure and so the dominant failure mode is by matrix cracking.

The exact location where the maximum value of the transverse tensile failure index occurs along the ply is highlighted in Fig. 12. Also, the distribution of the failure index in the straight-fiber model is compared with that of the curvilinear-fiber model at the start of the snap-through step. As expected, the abrupt change in the orientation angle of the fibers that occurs in the second and fourth plies of the straight-fiber model leads to high transverse stress gradients at the boundary between the symmetric and unsymmetric regions and so the maximum transverse tensile failure index is located there. In the curvilinear-fiber model, however, the smooth variation of fiber orientation angle allows for a more uniform transverse stress distribution along the same plies and so the maximum transverse tensile failure index is now transferred to the free-edge of the symmetric region. 
Table 5. Maximum tensile failure indices measured in the straight fiber model during the snap-through stage.

\begin{tabular}{|l|c|c|}
\cline { 2 - 3 } \multicolumn{1}{c|}{} & Transverse & Longitudinal \\
\hline Maximum failure index & $3.46 \times 10^{-1}$ & $4.54 \times 10^{-2}$ \\
Ply number & 4 & 1 \\
Displacement [mm] & 0.0 & 19.5 \\
Reaction Force [N] & 0.0 & 5.1 \\
\hline
\end{tabular}

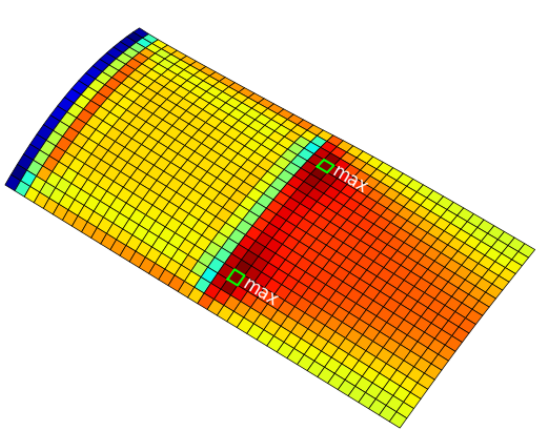

a)

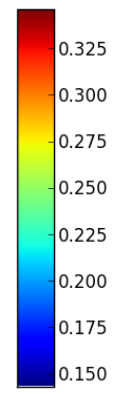

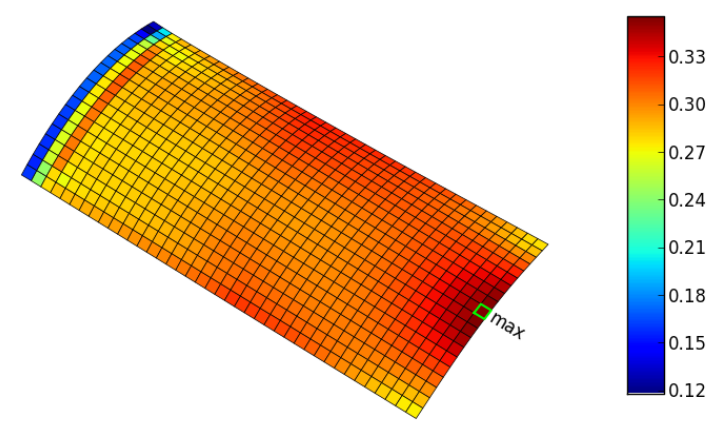

b)

Figure 12. Perspective view of the laminate models at the start of the snap-through. The color map represents the value of the transverse tensile failure index of each element. a) Straight fiber model. b) Curvilinear fiber model.

Fig. 13 shows the distribution of longitudinal tensile failure index along the bottom ply of the straight-fiber matrix for the load increment where this failure index is maximum. This load increment corresponds to the second critical point of the snap-through process, as can be seen in Fig. 11. It can be observed that the elements where the longitudinal tensile failure index is nonzero are mainly located near the free-edge of the unsymmetric region, where the curvature is changing sign due to the buckling of the unsymmetric region.

Table 6 gives the values of the maximum transverse tensile and longitudinal tensile failure indices for the curvilinear fiber model and when snapping from the second to the first shape. Similarly to the straight-fiber model, it is verified that both transverse and longitudinal compressive failure indices are zero over the curvilinear fiber plate during the whole snap-through stage and that the transverse tensile is higher than the longitudinal tensile failure index. The value of maximum transverse tensile failure index is about $7 \%$ higher than the that of the straight-fiber model while the value of maximum longitudinal tensile failure index is about $11 \%$ lower than the that of the straight-fiber model.

Fig. 14 shows the distribution of longitudinal tensile failure index over the straight and curvilinear-fiber plates at the start of the snap-through step. For both models, the maximum failure index is located at the top ply and at the middle of the free-edge of the unsymmetric region, where a high gradient of the the failure index is observed. Also nonzero longitudinal tensile failure index is verified only at the elements of the symmetric region of the fourth ply for both plates.

Fig. 15 shows the distribution of transverse tensile failure index over the bottom ply of the curvilinear-fiber plate at the load increment where the maximum failure index was verified. This load 


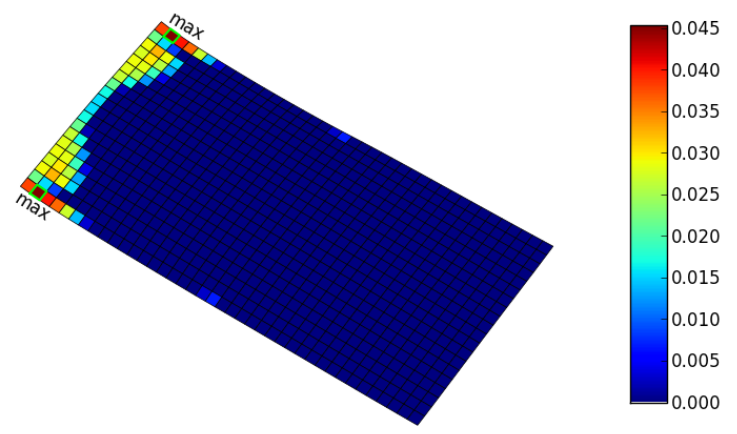

Figure 13. Perspective view of the straight-fiber plate model for maximum longitudinal tensile failure index. The color map represents the value of the longitudinal tensile failure index of each element.

Table 6. Maximum tensile failure measured in the curvilinear-fiber model during the snap-through step.

\begin{tabular}{|l|c|c|}
\cline { 2 - 3 } \multicolumn{1}{c|}{} & Transverse & Longitudinal \\
\hline Maximum failure index & $3.71 \times 10^{-1}$ & $4.02 \times 10^{-2}$ \\
Ply number & 1 & 4 \\
Displacement [mm] & 31.1 & 0.0 \\
Reaction Force [N] & 2.8 & 0.0 \\
\hline
\end{tabular}

increment corresponds to point $b$ in Fig. 11. Thus, at the second critical point, the straight-fiber model reaches the maximum longitudinal tensile failure index while the curvilinear-fiber model reaches the maximum transverse tensile failure index. This point corresponds to the second snap-through event when the unsymmetric region of the plates completely inverts its curvature to yield the first shape. The maximum value of the transverse tensile failure index is located at the free-edge of the unsymmetric region close to the point where the curvature of the free edge inverts sign.

\section{CONCLUDING REMARKS}

This paper presents a strategy to combine in a single laminate the multistability properties of thin unsymmetric laminates with the characteristics of Variable Stiffness Plates. The resulting laminate may be manufactured with AFP technology. However, this model introduces some simplifications that should be taken into account. This simplifications are concerned with tow drops and overlaps that will occur when placing finite width tows into a flat tool surface. In addition, the limitation that arises for small fiber turning radius should be investigated for the configuration adopted for the Variable Stiffness plies.

The multi-stable Variable Stiffness plate studied in this paper is composed of two regions with different stacking sequences. One of the regions develops significant curvature during the cure process while the other deforms only slightly. This may simulate the interaction between a moving component and a stiff structure and thus may be applied to morphing structures. For this purpose, it is essential that proper actuation mechanisms are included in the structure, which may be a subject of further research. 


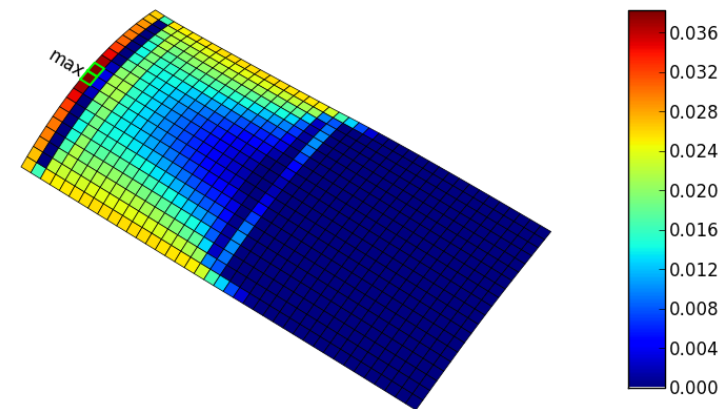

a)

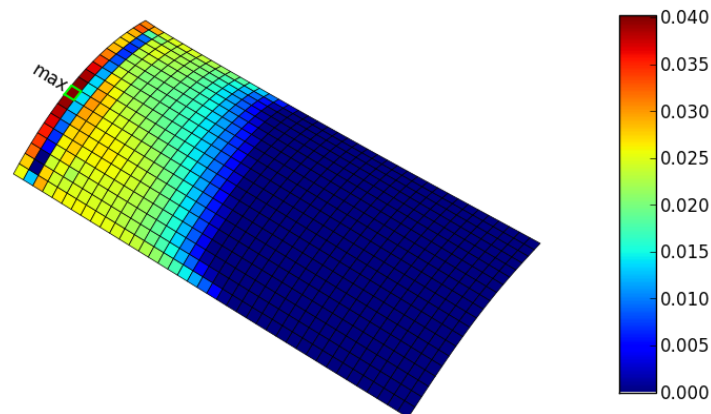

b)

Figure 14. Perspective view of the laminate models at the start of the snap-through. The color map represents the value of the longitudinal tensile failure index of each element. a) Straight fiber model. b) Curvilinear fiber model.

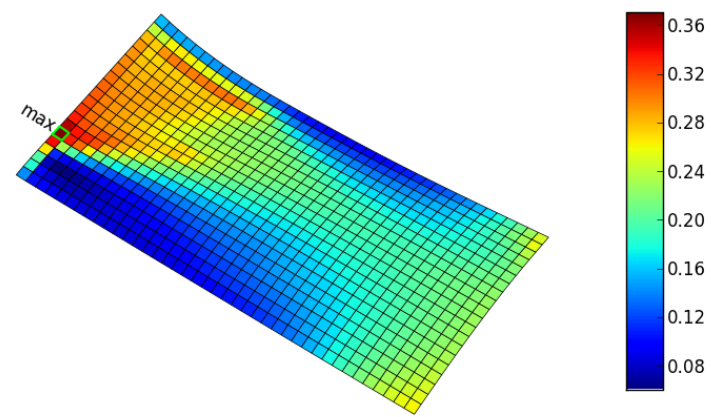

Figure 15. Perspective view of the curvilinear-fiber plate model for maximum transverse tensile failure index. The color map represents the value of the failure index of each element.

A Finite Element model was developed to analyse the variation of displacements and curvature of the multi-stable Variable Stiffness laminate during the cool-down of the laminate. The shapes obtained after the cool-down were compared with a similar model but composed of straight-fiber plies. The Variable Stiffness laminate preserves the bi-stable characteristics of the straigh-fiber panel. However the curvature characteristics of these two models are different. The variable stiffness model presents more curvature in the second stable shape than the straight-fiber model. However, the unsymmetric region of the variable stiffness laminate exhibits considerably less curvature for the first stable shape. This as direct effect on the critical loads required for changing from one shape to the other. The equilibrium paths of both straight and Variable Stiffness laminates during the snap-through step were predicted using two solution methods. The modified RIKS method predicted a lower critical load for the snap-through of the Variable Stiffness laminate from the second to the first shape in comparison with the load required to snap the straight-fiber laminate. This may be beneficial for some applications, since this decrease in critical load may be translated in less actuation energy, but may also be undesired for other applications that rely on this type of structures to withstand high surface 
pressure loads.

A first-ply failure analysis of the laminate was performed using the LaRC03 failure criteria. It was verified that both laminates can carry the loads without the onset of failure during the snap-through step. In addition, the LaRC03 criteria predicts that the main failure mode during this step is by matrix cracking for both straight and variable stiffness models. Moreover, the Variable Stiffness laminate has a slightly higher maximum transverse tensile failure index than the straight-fiber laminate. However, it was verified that removing the abrupt transition between symmetric and unsymmetric regions through the introduction of curvilinear-fiber plies may ameliorate the high stress gradients that occur at the interface leading to lower transverse tensile failure indices in this region. The smooth variation of fiber orientation angle over each ply ensures that the maximum value of transverse tensile stress occurs at a free-edge of the symmetric region instead of at the interface between unsymmetric and symmetric regions.

Several different configurations are possible for the description of the curvilinear-fibers that form the Variable Stiffness plies. This configurations may be obtained by varying three angle parameters as well as the stacking sequence of the laminate. The relationship between this parameters and the stable shapes obtained after cure, as well as its buckling behaviour is not yet fully understood and may raise a topic of further investigation.

\section{ACKNOWLEDGEMENTS}

This work was supported by the research grant PTDC/EME-PME/64819/2006, which was sponsored by the Fundação para a Ciência e a Tecnologia/Ministério da Ciência, Tecnologia e Ensino Superior.

The authors would like to thank the use of the services and facilities of the Porto University and of the Instituto Superior Técnico - Technical University of Lisbon. 


\section{A. GEOMETRY PLOTS}
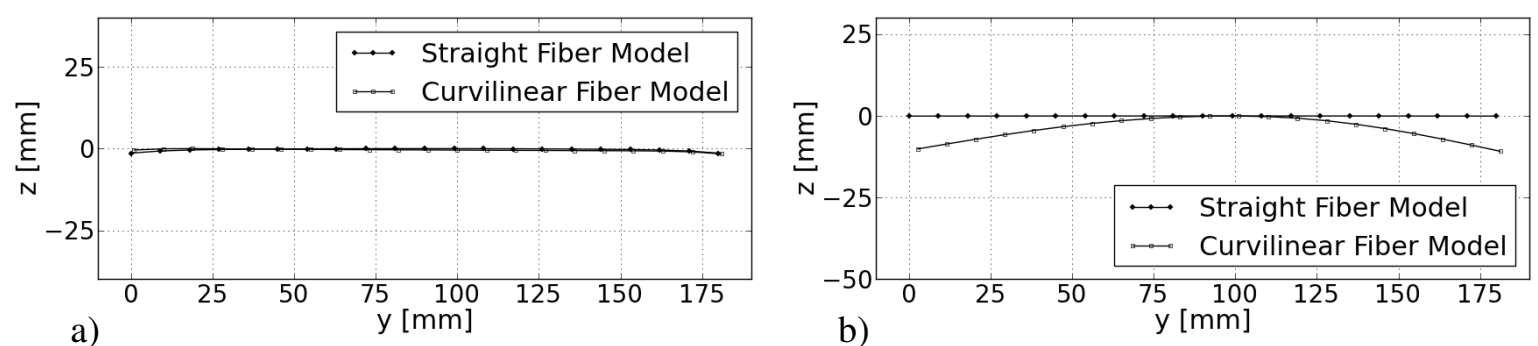

Figure 16. Comparison of the free-edges of the first shape between the straight and curvilinear fiber models. a) Free-edge at $x=0$. b) Free-edge at $x=360 \mathrm{~mm}$.
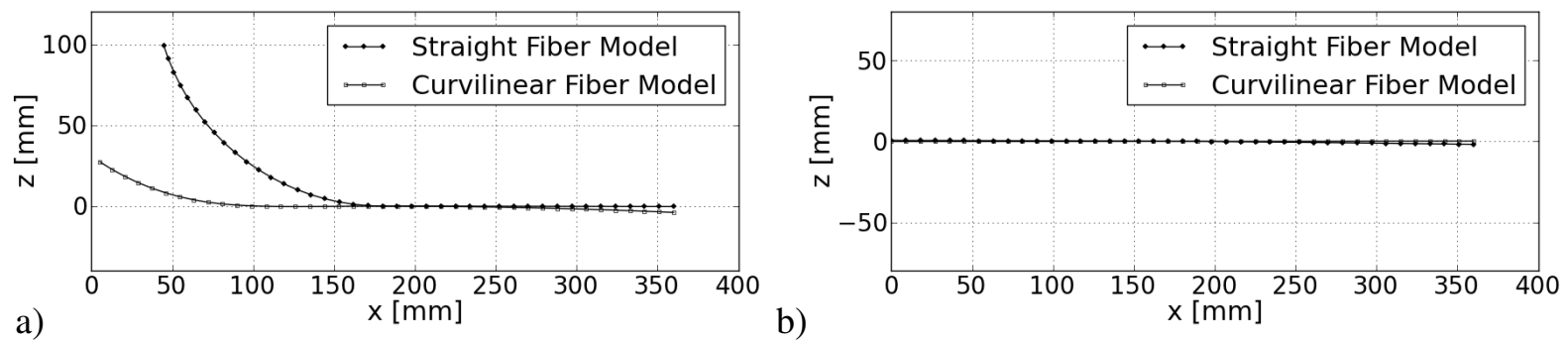

Figure 17. Longitudinal cross-section of deformed plate models. Comparison between the straight and curvilinear fiber models. a) First shape. b) Second shape.

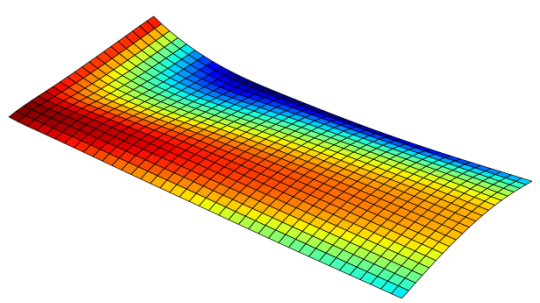

Figure 18. Perspective view of curvilinear fiber model at point $b$ in Fig. $10 p$. The color map represents the height of each point in the surface. 


\section{B. THE LARC FAILURE CRITERIA}

This appendix gives a description of the formulation of the LaRC failure criteria, [32, 33] .

\section{B.1. Transverse fracture}

The transverse fracture is associated with matrix fracture. The equations for transverse fracture are given in the following points.

\section{B.1.1 Tension}

The LaRC03 criteria used for predicting matrix cracking under transverse tension $\left(\sigma_{22} \geq 0\right)$ and in plane shear, are defined as:

$$
\begin{aligned}
& (1-g) \frac{\sigma_{22}}{Y_{T}}+g\left(\frac{\sigma_{22}}{Y_{T}}\right)^{2}+\left(\frac{\sigma_{12}}{S_{L}}\right)^{2}-1 \leq 0 \\
& (1-g) \frac{\sigma_{22}^{(m)}}{Y_{T}}+g\left(\frac{\sigma_{22}^{(m)}}{Y_{T}}\right)^{2}+\left(\frac{\sigma_{12}^{(m)}}{S_{L}}\right)^{2}-1 \leq 0, \\
& \sigma_{11}<0, \quad\left|\sigma_{11}\right|<X_{C} / 2
\end{aligned}
$$

where $Y_{T}$ is the in-situ transverse tensile strength, $X_{C}$ is the longitudinal compressive strength, $S_{L}$ is the in-situ shear strength, $g$ is the fracture toughness ratio defined as

$$
g=\frac{G_{I c}}{G_{I I c}}
$$

where $G_{I c}$ and $G_{I I c}$ are the mode I and II fracture toughness, respectively. The stress tensor components $\sigma_{i j}^{(m)}$ represent the components of the stress tensor in a frame aligned with the fiber direction:

$$
\begin{aligned}
& \sigma_{11}^{(m)}=\sigma_{11} \cos ^{2} \varphi+\sigma_{22} \sin ^{2} \varphi+2\left|\sigma_{12}\right| \sin \varphi \cos \varphi \\
& \sigma_{22}^{(m)}=\sigma_{11} \sin ^{2} \varphi+\sigma_{22} \cos ^{2} \varphi-2\left|\sigma_{12}\right| \sin \varphi \cos \varphi \\
& \sigma_{12}^{(m)}=-\sigma_{11} \sin \varphi \cos \varphi+\sigma_{22} \sin \varphi \cos \varphi+\left|\sigma_{12}\right|\left(\cos ^{2} \varphi-\sin ^{2} \varphi\right)
\end{aligned}
$$

where $\varphi$ is the fiber misalignment angle and represents the rotation of the fibers associated with an initial misalignement and with the stress tensor and is given by

$$
\begin{aligned}
\varphi & =\frac{\left|\sigma_{12}\right|+\left(G_{12}-X_{C}\right) \varphi^{c}}{G_{12}+\sigma_{11}-\sigma_{22}} \\
\varphi^{c} & =\tan ^{-1}\left[\frac{1-\sqrt{1-4\left(\frac{S_{L}}{X_{C}}+\eta^{L}\right) \frac{S_{L}}{X_{C}}}}{2\left(\frac{S_{L}}{X_{C}}+\eta^{L}\right)}\right]
\end{aligned}
$$

where $G_{12}$ is the shear modulus of the ply. The parameter $\eta^{L}$ is the coefficient of longitudinal friction defined by

with $\alpha_{0} \approx 53^{\circ}$.

$$
\eta^{L}=-\frac{S_{L} \cos 2 \alpha_{0}}{Y_{C} \cos ^{2} \alpha_{0}}
$$




\section{B.1.2 Compression}

The failure criteria used to predict matrix cracking under transverse compression $\left(\sigma_{22}<0\right)$ and in-plane shear are defined as:

$$
\begin{aligned}
& \left(\frac{\tau_{e}^{T}}{S_{T}}\right)^{2}+\left(\frac{\tau_{e}^{L}}{S_{L}}\right)-1 \leq 0, \\
& \sigma_{11} \geq-Y_{C} \\
& \left(\frac{\tau_{e}^{(m) T}}{S_{T}}\right)^{2}+\left(\frac{\tau_{e}^{(m) L}}{S_{L}}\right)-1 \leq 0, \\
& \sigma_{11} \leq-Y_{C}
\end{aligned}
$$

The effective shear stresses in the fracture plane are defined as:

$$
\begin{array}{r}
\tau_{e}^{T}=\left\langle\left|\tau^{T}\right|+\eta^{T} \sigma_{n} \cos \theta\right\rangle \\
\tau_{e}^{L}=\left\langle\left|\tau^{L}\right|+\eta^{L} \sigma_{n} \sin \theta\right\rangle
\end{array}
$$

where $\langle x\rangle$ is the McAuley operator defined as $\langle x\rangle=\frac{1}{2}(x+|x|)$. The angle $\theta$ is given by

$$
\theta=\tan ^{-1}\left(\frac{-\left|\sigma_{12}\right|}{\sigma_{22} \sin \alpha}\right)
$$

where the angle $\alpha$ is defined by the plane perpendicular to the laminate midplane and the fracture plane.

The components of the stress tensor on the fracture plane are given by:

$$
\left\{\begin{array}{l}
\sigma_{n}=\sigma_{22} \cos ^{2} \alpha \\
\tau^{T}=-\sigma_{22} \sin \alpha \cos \alpha \\
\tau^{L}=\sigma_{12} \cos \alpha
\end{array}\right.
$$

The terms $\tau^{(m) T_{e}}$ and $\tau_{e}^{(m) L}$ are calculated from equations (16) - (17) using the relevant components of the stress tensor established in a frame representing the fiber misalignment. The determination of $\alpha$ is performed numerically, in the failure criteria LaRC03, maximizing equations (14) and (15).

The coefficient of transverse influence, $\eta^{T}$, is obtained as:

$$
\eta^{T}=-\frac{1}{\tan 2 \alpha_{0}}
$$

\section{B.2. Longitudinal Failure}

\section{B.2.1 Tension}

The failure criteria used to predict fiber fracture under longitudinal tension $\left(\sigma_{11} \geq 0\right)$ is defined as:

$$
\frac{\sigma_{11}}{X_{T}}-1 \leq 0
$$




\section{B.2.2 Compression}

The failure criteria used to predict fiber fracture under longitudinal compression $\left(\sigma_{11}<0\right)$ and in-plane shear (fiber kinking) is established as a function of the components of the stress tensor in the rotating frame, defined in equation (10).

The criteria for fiber kinking are:

$$
\begin{aligned}
& \left\langle\frac{\left.\left|\sigma_{12}^{(m)}\right|+\eta^{L} \sigma^{(} m\right)_{22}}{S_{L}}\right\rangle-1 \leq 0, \\
& \sigma_{22}^{(m)}<0 \\
& (1-g) \frac{\sigma_{22}^{(m)}}{Y_{T}}+g\left(\frac{\sigma_{22}^{(m)}}{Y_{T}}\right)^{2}+\left(\frac{\sigma_{12}^{(m)}}{S_{L}}\right)^{2}-1 \leq 0, \\
& \sigma_{22}^{(m)} \geq 0, \quad\left|\sigma_{11}\right| \geq X_{C} / 2
\end{aligned}
$$




\section{References}

[1] Gibson, R. F., "A review of recent research on mechanics of multifunctional composite materials and structures," Composite Structures, Vol. 92, No. 12, pp. 2793-2810, 2010.

[2] McGowan, A.-M. R., Washburn, A. E., Horta, L. G., Bryant, R. G., Cox, D. E., Siochi, E. J., Padula, S. L., and Holloway, N. M., "Recent results from nasa's morphing project," in Smart Structures and Materials 2002, Proceedings of SPIE - The Int. Soc. for Opt. Eng., Vol. 4698, No. 97, San Diego, CA, 2002.

[3] Iannucci, L. and Fontanazza, L., "Design of morphing wing structures," in $3^{r} d$ SEAS DTC Technical Conference, Edinburgh, 2008.

[4] Thill, C., Etches, J. A., Bond, I. P., Potter, K. D., and Weaver, P. M., "Morphing skins," The Aeronautical Journal, Vol. 112, No. 1129, pp. 117-139, 2008.

[5] Sofla, A., Meguid, S., Tan, K., and Yeo, W., "Shape morphing of aircraft wing: Status and challenges," Materials and Design, Vol. 31, No. 3, pp. 1284-1292, 2010.

[6] Diaconu, C. G., Weaver, P. M., and Mattioni, F., "Concepts for morphing airfoil sections using bi-stable laminated composite structures," Thin-Walled Structures, Vol. 46, No. 6, pp. 689-701, 2008.

[7] Daynes, S., Weaver, P. M., and Potter, K. D., "Aeroelastic study of bistable composite airfoils," Journal of Aircraft, Vol. 46, No. 6, pp. 2169-2174, 2009.

[8] Mattioni, F., Weaver, P. M., Potter, K. D., and Friswell, M. I., "The application of thermally induced multistable composites to morphing aircraft structures," in Proceedings of SPIE, Vol. 6930, 693012, San Diego, CA, Mar. 2008.

[9] Lachenal, X., Daynes, S., and Weaver, P. M., "Review of morphing concepts and materials for wind turbine blade applications," Wind Energy, 2012.

[10] Lachenal, X., Weaver, P. M., and Daynes, S., "Multi-stable composite twisting structure for morphing applications," Proceedings of the Royal Society A, Vol. 468, No. 2141, pp. 1230-1251, May 2012.

[11] Portela, P., Camanho, P., Weaver, P., and Bond, I., "Analysis of morphing, multi stable structures actuated by piezoelectric patches," Computers and Structures, Vol. 86, No. 3-5, pp. 347-356, 2008 .

[12] Correia, V. M. F., Gomes, M. A. A., Suleman, A., Soares, C. M. M., and Soares, C. A. M., "Modelling and design of adaptive composite structures," Computational Methods in Applied Mechanics and Engineering, Vol. 185, pp. 325-346, 2000.

[13] Mattioni, F., Weaver, P., Potter, K., and Friswell, M., "Analysis of thermally induced multistable composites," International Journal of Solids and Structures, Vol. 45, No. 2, pp. 657-675, 2008.

[14] Mattioni, F., Weaver, P., and Friswell, M., "Multistable composite plates with piecewise variation of lay-up in the planform," International Journal of Solids and Structures, Vol. 46, No. 1, pp. 151-164, 2009.

[15] Hyer, M. W., "Some observations on the cured shape of thin unsymmetric laminates," Journal of Composite Materials, Vol. 15, pp. 175-194, Mar. 1981. 
[16] Hamamoto, A. and Hyer, M. W., "Non-linear temperature-curvature relationships for unsymmetric graphite-epoxy laminates," International Journal of Solids and Structures, Vol. 23, No. 7, pp. 919-935, 1987.

[17] Dano, M.-L. and Hyer, M. W., "Thermally-induced deformation behavior of unsymmetric laminates," International Journal of Solids and Structures, Vol. 35, No. 17, pp. 2101-2120, 1998.

[18] Dano, M.-L. and Hyer, M. W., "The response of unsymmetric laminates to simple applied forces," Mechanics of Composite Materials and Structures, Vol. 3, No. 1, pp. 65-80, 1996.

[19] Dano, M.-L. and Hyer, M. W., "Snap-through of unsymmetric fiber-reinforced composite laminates," International Journal of Solids and Structures, Vol. 39, No. 1, pp. 175-198, 2002.

[20] Potter, K., Weaver, P., Seman, A. A., and Shah, S., "Phenomena in the bifurcation of unsymmetric composite plates," Composites Part A: Applied Science and Manufacturing, Vol. 38, No. 1, pp. 100-106, 2007.

[21] Pirrera, A., Avitabile, D., and Weaver, P., "Bistable plates for morphing structures: A refined analytical approach with high-order polynomials," International Journal of Solids and Structures, Vol. 47, No. 25-26, pp. 3412-3425, 2010.

[22] Gürdal, Z. and Olmedo, R., "Composite laminates with spatially varying fiber orientations: Variable stiffness panel concept," in Proceedings of the $33^{\text {rd }}$ AIAA/ASME/ASCE/AHS/ASC Structures, Structural Dynamics, and Materials (SDM) Conference, Dallas, TX, Apr. 1992, pp. 798-808.

[23] Gürdal, Z. and Olmedo, R., "In-plane response of laminates with spatially varying fiber orientations: Variable stiffness concept," AIAA, Vol. 31, No. 4, pp. 751-758, Apr. 1993.

[24] Olmedo, R. and Gürdal, Z., "Buckling response of laminates with spatially varying fiber orientations," in Proceedings of the $34^{\text {th }}$ AIAA/ASME/ASCE/AHS/ASC Structures, Structural Dynamics, and Materials (SDM) Conference, La Jolla, CA, Apr. 1993, paper AIAA no. 93-1567, pp. 22612269.

[25] Wu, K. C., Thermal and structural performance of tow-placed, variable stiffness panels. IOS Press, Sep. 2006.

[26] Abaqus/CAE, User's Manual. Dassault Systemes Simulia Corp., Providence, RI, USA, 2010.

[27] Waldhart, C. J., "Analysis of tow-placed, variable-stiffness laminates," Master's thesis, Virginia Polytechnic Institute and State University, Blacksburg, VA, Jun. 1996.

[28] Hahn, H. T. and Pagano, N. J., "Curing stresses in composite laminates," Journal of Composite Materials, Vol. 9, No. 1, pp. 91-106, Jan. 1975.

[29] Hyer, M. W., "The room-temperature shapes of four-layer unsymmetric cross-ply laminates," Journal of Composite Materials, Vol. 16, pp. 318-340, Jul. 1982.

[30] Crisfield, M. A., "A fast incremental/iterative solution procedure that handles 'snap-through'," Computers and Structures, Vol. 13, No. 1-3, pp. 55-62, 1981.

[31] Ramm, E., Strategies for tracing the nonlinear response near limit points, E. Wunderlich, E. S. and Bathe, K. J., Editors. Berlin: Springer-Verlag, 1981.

[32] Dávila, C. G., Camanho, P. P., and Rose, C. A., "Failure criteria for FRP laminates," Journal of Composite Materials, Vol. 39, pp. 323-345, 2005.

[33] Dávila, C. G. and Camanho, P. P., "Failure criteria for FRP laminates in plane stress," National Aeronautics and Space Administration, NASA/TM-2003-212663, 2003. 